\title{
CONHECIMENTO DAS FAMÍLIAS DE PACIENTES COM TUBERCULOSE SOBRE A DOENÇA: ESTUDO TRANSVERSAL*
}

\author{
Ana Angélica Rêgo de Queiroz ${ }^{1}$, Dândara Nayara Azevêdo Dantas², Rayssa Horácio Lopes ${ }^{3}$, Rejane Maria \\ Paiva de Menezes ${ }^{4}$, Bertha Cruz Enders 5 , Ricardo Alexandre Arcêncio ${ }^{6}$
}

RESUMO: Objetivou-se analisar o conhecimento dos familiares dos pacientes com tuberculose sobre a doença. Estudo epidemiológico descritivo de corte transversal realizado com a aplicação de questionário a 50 familiares de pacientes diagnosticados com tuberculose em Natal - RN entre os meses março e agosto de 2012. Os dados foram analisados através de frequências, percentuais, medidas de tendência central e cruzamento de variáveis dicotômicas com os testes Qui-Quadrado e teste de Fisher. Dentre os entrevistados, 34 (68\%) disseram possuir pouco conhecimento e $11(22 \%)$ nenhum conhecimento sobre a tuberculose. Quanto ao tempo de transmissão da doença, $20(40 \%)$ deram uma resposta errada. Conclui-se que o conhecimento expresso pelos familiares sobre a tuberculose em alguns aspectos, foi satisfatório. Entretanto, suscitou características preocupantes em relação. Frente a esse cenário é imprescindível a promoção e disseminação da informação sobre a doença, como forma de controle da tuberculose.

DESCRITORES: Tuberculose; Conhecimento; Família; Enfermagem.

\section{KNOWLEDGE OF THE FAMILIES OF PATIENTS WITH TUBERCULOSIS REGARDING THIS DISEASE: A TRANSVERSAL STUDY}

\begin{abstract}
The article aimed to analyze the knowledge of the family members of the patients with tuberculosis regarding this disease. It is an epidemiological, descriptive and transversal study undertaken through a questionnaire applied to 50 family members of patients diagnosed with tuberculosis in Natal (State of Río Grande do Norte - RN) between March and August 2012. The data were analyzed through frequencies, percentages, measurements of central tendency and the combining of dichotomous variables using the Chi-squared test and Fisher's exact test. Among the interviewees, $34(68 \%)$ stated that they had little knowledge, and 11 (22\%) that they had no knowledge, regarding tuberculosis. In relation to the infectious period of the disease, 20 (40\%) gave incorrect responses. It is concluded that the knowledge expressed by the family members regarding tuberculosis was satisfactory in certain aspects. However, it raised worrying characteristics in relation to the disease. In this scenario, it is essential to promote and disseminate information regarding the disease, as a form of controlling tuberculosis.
\end{abstract}

DESCRIPTORS: Tuberculosis; Knowledge; Family; Nursing.

\section{CONOCIMIENTO DE LAS FAMILIAS DE PACIENTES CON TUBERCULOSIS ACERCA DE LA ENFERMEDAD: ESTUDIO} TRANSVERSAL

RESUMEN: El objetivo del estudio fue analizar el conocimiento de los familiares de pacientes con tuberculosis acerca de la enfermedad. Estudio epidemiológico descriptivo transversal realizado por medio de cuestionario a 50 familiares de pacientes con diagnóstico de tuberculosis en Natal-RN, entre los meses de marzo y agosto de 2012. Los datos fueron analizados considerándose frecuencias, porcentuales, medidas de tendencia central y cruzamiento de variables dicotómicas con los testes Chi-Cuadrado y test de Fisher. Entre los entrevistados, 34 (68\%) afirmaron tener poco conocimiento y $11(22 \%)$ ningun conocimiento sobre tuberculosis. Cuanto al tiempo de transmisión de la enfermedad, 20 (40\%) contestaron equivocadamente. Se concluye que el conocimiento expreso por los familiares sobre la tuberculosis en algunos aspectos fue satisfactorio. Sin embargo, se revelaron características preocupantes acerca de eso. Delante del cuadro, son imprescindibles la promoción y divulgación de la información acerca de la enfermedad, como forma de control de la tuberculosis.

DESCRIPTORES: Tuberculosis; Conocimiento; Familia; Enfermería.

*Artigo extraído da dissertação intitulada: "O conhecimento e as atitudes das famílias de pacientes em tratamento da tuberculose na Atenção Primária à Saúde." Universidade Federal do Rio Grande do Norte, 2012.

${ }^{1}$ Enfermeira. Mestre em Enfermagem. Doutoranda do Programa Interunidades de Doutoramento em Enfermagem da Escola de Enfermagem de Ribeirão Preto, Universidade de São Paulo. Ribeirão Preto, SP, Brasil.

${ }^{2}$ Enfermeira. Mestre em Enfermagem. Docente do Departamento de Enfermagem da Universidade Federal do Rio Grande do Norte. Natal, RN, Brasil.

${ }^{3}$ Enfermeira. Mestre em Enfermagem. Docente da Escola Técnica de Enfermagem da Universidade Federal do Rio Grande do Norte. Natal, RN, Brasil.

${ }^{4}$ Enfermeira. Doutora em Enfermagem. Docente do Departamento de Enfermagem da Universidade Federal do Rio Grande do Norte. Natal, RN, Brasil.

${ }^{5}$ Enfermeira. PhD em Enfermagem. Docente do Programa de Pós-Graduação em Enfermagem da Universidade Federal do Rio Grande do Norte. Natal, RN, Brasil.

${ }^{6}$ Enfermeiro. Doutor em Enfermagem. Docente do Departamento Enfermagem Materno-Infantil e Saúde Pública da Escola de Enfermagem de Ribeirão Preto, Universidade de São Paulo. Ribeirão Preto, SP, Brasil.

Autor Correspondente:

Ana Angélica Rêgo de Queiroz

Recebido: 16/07/2015

Universidade de São Paulo

Av. dos Bandeirantes, 3900 - 14040-902 - Ribeirão Preto, SP, Brasil

Email: aninha_arego@hotmail.com 


\section{- INTRODUÇÃO}

A tuberculose (TB) representa ainda no século XXI um desafio para as autoridades sanitárias no Brasil e no mundo pela alta incidência de mortalidade entre as doenças infectocontagiosas. Dentre os 22 países que concentram cerca de $80 \%$ dos casos de tuberculose, o Brasil ocupa a $16^{a}$ posição em relação ao número de casos novos e a $22^{\mathrm{a}}$ posição em relação ao coeficiente de incidência, prevalência e mortalidade. No ano de 2014 o coeficiente de incidência foi de 33,5 casos por 100.000 habitantes e a taxa de mortalidade (considerando os casos encerrados em 2013) foi de 2,3 óbitos por 100.000 habitantes, sendo assim, considerado como um dos países prioritários para o controle da doença ${ }^{(1)}$.

O desconhecimento por parte dos familiares, no que se refere à doença ou enfermidade de modo geral, a evolução do quadro clínico, as possíveis complicações, a forma adequada de cuidar no domicílio e as demandas físicas e sociais, provoca uma velada inquietação ${ }^{(2)}$.

Destarte, a investigação dos fatores associados ao conhecimento das famílias sobre a TB é necessária para identificar os principais obstáculos ao sucesso do tratamento e dos grupos prioritários do ponto de vista de um acompanhamento diferenciado. A família se constitui como fator relevante que deve ser levado em consideração no controle da TB. O surgimento dessa doença no âmbito familiar causa impacto nas relações entre seus membros no que se refere ao modo de enfrentá-la.

Essas alterações de comportamento individuais e familiares estão diretamente relacionadas ao conhecimento e atitudes em relação à enfermidade ${ }^{(3)}$. Conhecimentos insuficientes podem afetar a forma de percebê-la e de enfrentá-la, portanto, podem potencializar a vulnerabilidade à TB.

Diante disso, o conhecimento torna-se um alvo de estratégias essenciais para a formulação de políticas de saúde efetivas que objetivem o controle da doença tendo a família como um objeto importante do processo de trabalho da enfermagem na Atenção Primária à Saúde ${ }^{(3)}$.

Torna-se indispensável informar esses sujeitos quanto à enfermidade e resgatar os conhecimentos em relação ao processo saúde-doença de maneira que se promovam a desmistificação e redução do estigma social de doenças como a TB. Além disso, deve-se levar em consideração que o conhecimento pode influenciar as práticas em relação à prevenção.

Compreender a família como a mais constante unidade de saúde é condição sine qua non para a instituição de uma realidade sanitária, para a promoção, prevenção, tratamento e reabilitação. De acordo com o conceito de unidade de cuidado, a família é definida pelos seus próprios membros. Nesse processo de cuidar, a família se preocupa não somente com o estado de saúde físico do indivíduo doente, mas com seu bem-estar e felicidade ${ }^{(4)}$.

Nesse estudo, considera-se conhecimento como a lembrança de acontecimentos ou fatos transmitidos pelo sistema de educação ao qual o sujeito participa, emitindo conceitos com o entendimento adquirido sobre determinado assunto ou evento ${ }^{(5)}$. Utilizou-se como referencial teórico norteador do desenvolvimento do presente estudo, a Atenção Primária à Saúde (APS), proposto por Starfield.

Esse estudo justifica-se a partir da transposição do enfoque da TB do individual para o familiar. Essa investigação irá contribuir para a reorganização dos processos de trabalho na Atenção Primaria à Saúde no contexto dessa doença para o âmbito coletivo, com elucidação e análise do conhecimento existente no ambiente familiar sobre a doença. Ademais, favorecerá mudanças de práticas que colaborem para o controle da doença e contribuam para o fortalecimento da APS como local importante para o controle da TB com auxílio na definição de ações prioritárias para redução de índices da doença nas comunidades.

Isto posto, objetiva-se com esse estudo analisar o conhecimento dos familiares dos pacientes com tuberculose sobre a doença. 


\section{- MÉTODO}

Trata-se de um estudo epidemiológico descritivo de corte transversal conduzido no município de Natal-RN com familiares e coabitantes de pacientes em tratamento de TB pulmonar na Atenção Primária à Saúde. A coleta de dados ocorreu entre primeiro de março e 31 de agosto de 2012.

Como critérios de inclusão no estudo, foram selecionados os sujeitos de ambos os sexos, comunicantes dos pacientes de TB, com idade igual ou maior a 18 anos na ocasião da coleta, residentes em Natal e com possibilidade de participação durante a entrevista. Foram excluídos o que não possuíam essas características. A amostragem foi consecutiva, sendo selecionados 50 sujeitos da população de referência do estudo.

Esses indivíduos foram recrutados com investigação dos enfermeiros das unidades municipais, que indicavam os pacientes em tratamento da TB pulmonar. Em seguida, identificou-se os familiares ou coabitantes de pacientes em tratamento da TB e estes foram convidados a participar da pesquisa com visitas domiciliárias, ou no momento da consulta marcada de retorno do acompanhamento do tratamento do doente.

O instrumento utilizado para coleta dos dados foi elaborado com base em estudos publicados sobre conhecimento e atitudes(6-8). O mesmo foi submetido à validade de face e de conteúdo por dez juízes, especialistas na temática e pré-testado em 10 membros familiares dos pacientes de TB. É composto por 63 itens que seguem predominantemente a escala de Likert de cinco pontos $(1=$ nunca; $2=$ quase nunca; 3 = às vezes; $4=$ quase sempre e $5=$ sempre), em que os participantes informaram a frequência com que percebiam a ocorrência de um dado evento. Além disso, possui questões relacionadas aos aspectos sociodemográficos, informações clínico-epidemiológicas, conhecimento sobre a doença e sobre as atitudes das famílias quanto à doença.

Para o desenvolvimento desse estudo, especificamente, foram utilizadas as variáveis: estado civil, idade, escolaridade, ocupação formal, religião, número de moradores por domicílio, salário, acesso à internet, hábito de ler revistas e jornais, assistir televisão relacionadas ao perfil sociodemográfico, conhecimento e atitude das famílias quanto à TB.

Após a aprovação do projeto pelo Comitê de Ética em Pesquisa da Universidade Federal do Rio Grande do Norte (UFRN) - Parecer N $N^{\circ} 323 / 2011$, a coleta de dados foi cumprida entre os meses de março e agosto de 2012.

Os dados coletados foram implantados no programa Excel, com dupla digitação independente. Após a verificação de erros e inconsistências, a análise foi realizada nos softwares STATISTICA 9.0 da StatSoft e do SPSS para Mac. A pesquisa contemplou uma fase descritiva, com análise exploratória dos dados, sendo estimadas as frequências relativas, medidas de tendência central (média, mediana). Em seguida, foi realizada análise bivariada do cruzamento das variáveis dicotomizadas - conhecimento sobre TB e mudanças de atitudes com as variáveis independentes (idade, escolaridade, hábitos de leitura de jornal, revistas, e acesso à internet) por meio de tabelas de contingência, quando então aplicou-se o teste Qui-Quadrado e nos casos necessários o teste exato de Fisher. Nas tabelas 2x2 foi computado o Odds Ratio com respectivo intervalo de confiança de 95\% (IC 95\%). A probabilidade do erro tipo I aceito no estudo foi de até $5 \%$.

\section{RESULTADOS}

A Tabela 1 revela o perfil sociodemográfico dos sujeitos da investigação. Observou-se que 43 (86\%) dos entrevistados eram do sexo feminino, 34 (68\%) possuíam idade entre 30 e 59 anos, sendo a média da idade 46,64 anos e a mediana 46,50 anos. No que se refere à ocupação, 24 (48\%) eram do lar e tinham renda familiar mensal maior que $\mathrm{R} \$ 1.000,00$.

Quanto ao conhecimento dos familiares dos pacientes com TB sobre a doença, 34 (68\%) disseram possuir pouco conhecimento, $11(22 \%)$ afirmaram nenhum conhecimento. Entretanto, $31(62 \%)$ relataram saber alguma coisa sobre TB. Dos 50, 27 (54\%) dos entrevistados afirmaram que nunca procuram saber 
sobre informações sobre a tuberculose. Apesar disto, quando indagados se as informações acerca da TB costumam ser esclarecedoras nas situações em que procuram, 22 (44\%) responderam como satisfatório.

A Figura 1 apresenta a opinião dos sujeitos da pesquisa quanto aos sintomas que eles acreditam ser mais comuns da tuberculose. Dentre as respostas apresentadas, os sintomas reconhecidos foram febre, dor no peito e costas (46 sujeitos); seguidos por perda de peso (45 sujeitos), inapetência e tosse há mais de três semanas (41 sujeitos).

Em relação ao microorganismo causador da TB, $18(36 \%)$ sujeitos responderam de forma correta, afirmando que a doença era causada por bactéria, 17 (34\%) dos entrevistados disseram que não sabiam e 14 (28\%) responderam que a TB é causada por vírus e um (2\%) participante relatou o fungo como agente causal.

As formas de transmissão reconhecidas pelos familiares dos pacientes de TB estão destacadas na Figura 2. Pode-se constatar que 45 sujeitos apontaram que a TB é transmitida quando você está no mesmo ambiente do sintomático doente, 40 associaram à TB ao uso comum de pratos, talheres e copos.

Em relação ao conhecimento acerca da tuberculose, quando os sujeitos foram indagados a respeito do tempo em que uma pessoa com tuberculose pulmonar deixa de transmitir a doença, 25 (50\%) entrevistados responderam que não sabiam e 20 (40\%) deram uma resposta errada. Em relação à questão abordada, se após o início do tratamento o indivíduo deixa de transmitir a TB, 22 (44\%) referiram sim. No tocante ao tempo de tratamento, $40(80 \%)$ dos entrevistados responderam corretamente.

A Tabela 2 expressa a associação das variáveis estado civil, idade, escolaridade, ocupação, religião, número de moradores por domicílio, salário, acesso à internet, hábito de ler jornais e assistir televisão com o conhecimento sobre TB. Do total de variáveis independentes consideradas nesta etapa da análise, observou-se que a variável salário mínimo se apresentou com significância estatística $(p<0,05)$, o que demonstra associação da variável salário mínimo com o conhecimento sobre TB.
Tabela 1 - Perfil sociodemográfico dos familiares e o grau de parentesco com os pacientes em tratamento de tuberculose. Natal, RN, Brasil, 2012

\begin{tabular}{|c|c|c|}
\hline Variáveis & $\mathrm{n}=50$ & $\%$ \\
\hline \multicolumn{3}{|l|}{ Idade } \\
\hline$<30$ anos & 7 & 14 \\
\hline $30-59$ & 34 & 68 \\
\hline$\geq 60$ anos & 9 & 18 \\
\hline \multicolumn{3}{|l|}{ Sexo } \\
\hline Masculino & 7 & 14 \\
\hline Feminino & 43 & 86 \\
\hline \multicolumn{3}{|l|}{ Ocupação } \\
\hline Do lar & 24 & 48 \\
\hline Aposentado/Pensionista & 8 & 16 \\
\hline Desempregado & 4 & 8 \\
\hline Agente Comunitário de Saúde & 2 & 4 \\
\hline Autônomo & 10 & 20 \\
\hline Auxiliar de Serviços Gerais & 2 & 4 \\
\hline \multicolumn{3}{|l|}{ Renda } \\
\hline$<600,00$ & 7 & 14 \\
\hline $600,00-1.000,00$ & 18 & 36 \\
\hline$>1.000,00$ & 24 & 48 \\
\hline \multicolumn{3}{|l|}{ Escolaridade } \\
\hline Sem escolaridade & 6 & 12 \\
\hline Ensino fundamental & 25 & 50 \\
\hline Ensino médio & 16 & 32 \\
\hline Ensino superior & 3 & 6 \\
\hline \multicolumn{3}{|l|}{ Grau de Parentesco } \\
\hline Avós & 1 & 2 \\
\hline Mãe & 12 & 24 \\
\hline Pai & 1 & 2 \\
\hline Filho(a) & 7 & 14 \\
\hline Irmão(a) & 4 & 8 \\
\hline Neto(a) & 1 & 2 \\
\hline Primo & 1 & 2 \\
\hline Esposo(a) & 14 & 28 \\
\hline Outros & 9 & 18 \\
\hline
\end{tabular}




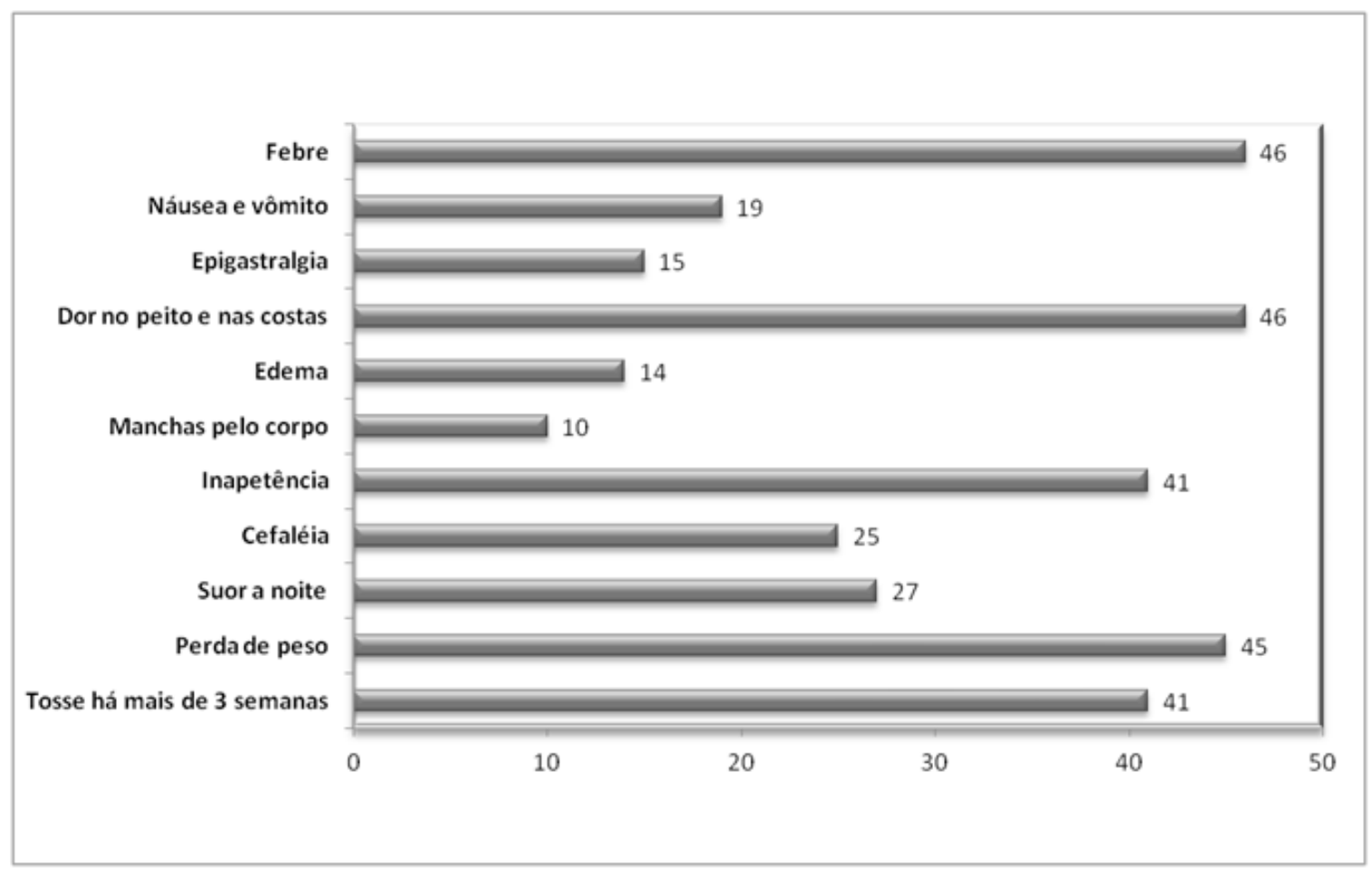

Figura 1 - Frequência absoluta dos sintomas comuns da tuberculose, apontados pelos familiares de pacientes em tratamento. Natal, RN, Brasil, 2012

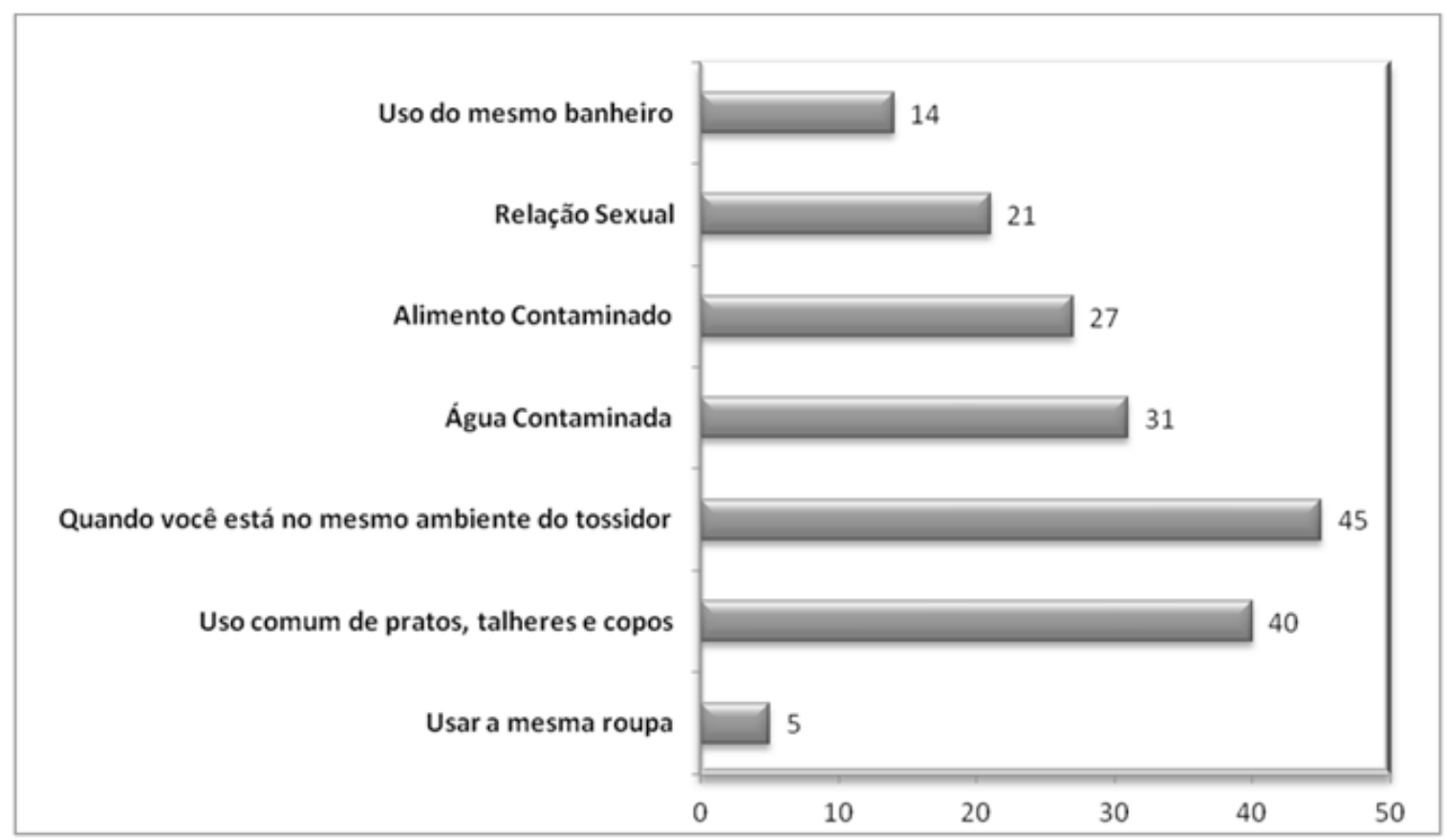

Figura 2 - Frequência absoluta das formas de transmissão da doença, reconhecidas pelos membros familiares de pacientes em tratamento da tuberculose. Natal, RN, Brasil, 2012 
Tabela 2 - Fatores associados ao conhecimento sobre Tuberculose em famílias de pacientes diagnosticados da doença na Atenção Primária à Saúde. Natal, RN, Brasil, 2012

\begin{tabular}{|c|c|c|c|c|c|c|c|}
\hline \multirow[t]{3}{*}{ Fatores Associados ao Conhecimento } & \multicolumn{4}{|c|}{ Conhecimento } & \multirow{3}{*}{$\frac{\text { ODDS }}{\text { Ratio }}$} & \multirow{3}{*}{ IC (95\%) } & \multirow{3}{*}{$\mathbf{p}$} \\
\hline & \multicolumn{2}{|c|}{ Não } & \multicolumn{2}{|c|}{ Sim } & & & \\
\hline & $\mathrm{n}$ & $\%$ & $\mathrm{n}$ & $\%$ & & & \\
\hline \multicolumn{8}{|l|}{ Estado civil casado: } \\
\hline Sim & 6 & 54,5 & 30 & 76,9 & 1 & - & \\
\hline Não & 5 & 45,5 & 9 & 23,1 & 2,778 & $0,684-11,27$ & 0,252 \\
\hline \multicolumn{8}{|l|}{ Idade } \\
\hline$<47,04$ & 7 & 63,6 & 18 & 46,2 & 1 & - & 0,306 \\
\hline$>47,04$ & 4 & 36,4 & 21 & 53,8 & 2,042 & $0,513-8,119$ & \\
\hline \multicolumn{8}{|l|}{ Escolaridade } \\
\hline Sim & 8 & 72,7 & 36 & 92,3 & 1 & - & \\
\hline Não & 3 & 27,3 & 3 & 7,7 & 0,222 & $0,038-1,310$ & 0,111 \\
\hline \multicolumn{8}{|l|}{ Ocupação Formal } \\
\hline Sim & 0 & 0 & 5 & 12,8 & 1 & - & \\
\hline Não & 11 & 100 & 34 & 87,2 & 1,324 & $1,121-1,563$ & 0,573 \\
\hline \multicolumn{8}{|l|}{ Número moradores domicílio } \\
\hline$<4$ & 3 & 27,3 & 9 & 23,1 & 1 & - & \\
\hline$>4$ & 8 & 72,7 & 30 & 76,9 & 1,25 & $0,273-5,725$ & 0,528 \\
\hline \multicolumn{8}{|l|}{ Salário } \\
\hline$>1,7$ S.M & 2 & 18,2 & 23 & 59 & 1 & & \\
\hline$<1,7$ S. M & 9 & 81,8 & 16 & 41 & 0,155 & $0,029-0,813$ & $0,017^{* *}$ \\
\hline \multicolumn{8}{|l|}{ Ler jornal } \\
\hline Sim & 4 & 36,4 & 18 & 46,2 & 1 & - & \\
\hline Não & 7 & 63,6 & 21 & 53,8 & 0,667 & $0,168-2,65$ & 0,734 \\
\hline \multicolumn{8}{|l|}{ Assistir TV } \\
\hline Sim & 8 & 72,7 & 31 & 79,5 & 1 & - & \\
\hline Não & 3 & 27,3 & 8 & 20,5 & 0,688 & $0,148-3,203$ & 0,688 \\
\hline \multicolumn{8}{|l|}{ Acesso à internet } \\
\hline Sim & 1 & 9,1 & 11 & 28,2 & 1 & - & \\
\hline Não & 10 & 90,9 & 28 & 71,8 & 0,255 & $0,029-2,231$ & 0,257 \\
\hline
\end{tabular}

\section{- DISCUSSÃO}

Propôs-se investigar o conhecimento das famílias de pacientes em tratamento da TB sobre a doença. A partir dos resultados, pode-se constatar a predominância do sexo feminino, o que pode ser justificado pelos dados do IBGE ${ }^{(9)}$, que apresenta as mulheres (53\%) da população de Natal. Além disso, estudos evidenciam que há uma provisão feminina no cuidado da família. Em uma pesquisa realizada em Ribeirão Preto (SP) com familiares de pacientes com TB, a maioria era do sexo feminino ${ }^{(10)}$.

No tocante à escolaridade, identificou-se que parte dos sujeitos apresentavam baixa escolaridade, o que remete a uma situação de vulnerabilidade social, que pode influenciar os indivíduos a não buscarem informações acerca da doença, bem como informações sobre saúde, de maneira geral. Além disso, pode ser o reflexo de um conjunto de condições socioeconômicas precárias, que aumentam a vulnerabilidade à tuberculose, sendo um fator de risco para a tuberculose, contribuindo inclusive para a não adesão ao tratamento e para o aumento da taxa de abandono ${ }^{(11,12)}$. 
O padrão de ocorrência da TB está relacionado aos determinantes sociais, que estão estruturados nos modos de produção e reprodução da sociedade, sendo as pessoas mais vulneráveis ao adoecimento as que estão entre as populações de baixa renda com condições insalubres de moradia ${ }^{(6)}$.

Ademais, no presente estudo observou-se um dado preocupante de que a falta de conhecimento sobre TB pode afetar a forma como a comunidade percebe e enfrenta a doença. Logo, é necessário resgatar os conhecimentos que a população detém sobre a saúde-doença para que se viabilize o planejamento de ações que promovam a desmistificação de enfermidades como a tuberculose e rupturas de preconceito e/ou estigma ${ }^{(13)}$.

No tocante aos sintomas da TB apontados, evidencia-se que as respostas foram satisfatórias, uma vez a maioria dos entrevistados afirmou a febre e dor no peito e nas costas como sintomas. Parte dos entrevistados também citou perda de peso, inapetência e tosse há mais de três semanas, além de cefaleia.

Em uma pesquisa realizada em um município do estado de São Paulo, a tosse por mais de três semanas, perda de peso e inapetência foram sintomas mais apresentados pelos sujeitos como sugestivos de tuberculose, enquanto que a sudorese noturna foi o sintoma menos reconhecido, embora $25 \%$ dos familiares não associassem a tosse crônica à $\mathrm{TB}^{(3)}$.

As manifestações clínicas da TB podem ser variadas. Os sintomas clássicos da TB pulmonar são: tosse persistente, produtiva ou não (com muco e eventualmente sangue), febre vespertina, sudorese noturna e emagrecimento ${ }^{(14)}$. Desta forma, percebe-se coerência nas respostas dos entrevistados quanto aos sintomas. Entretanto, percebeu-se que sintomas comumente citados na literatura, como a sudorese noturna, não foi indicado por um número relevante de entrevistados.

Observa-se também, que a quantidade dos participantes que responderam de forma errada, ou que afirmaram não saber a causa da TB foi bastante significativa. Corroborando com estudo realizado com estudantes sobre a temática, em relação à identificação da causa da enfermidade, a maioria dos estudantes $(56,6 \%)$ respondeu que também não sabia. E algumas pessoas que acreditavam conhecer a causa da enfermidade responderam da seguinte maneira: "doença produzida por vírus", "doença produzida por friagem" ou "complicação dos resfriados"(13).

No que se refere às formas de transmissão da doença, por se tratar de uma doença contagiosa, acredita-se que muitas pessoas generalizam as formas de contágio e creem que a TB pode também ser contraída por meio do uso de utensílios domésticos, do abraço e do aperto de mão. Em consonância com esses achados, estudo realizado com profissionais e estudantes de enfermagem no estado de São Paulo, verificou-se que parte dos sujeitos da referida pesquisa respondeu que se pode contrair a tuberculose, quando falar com as pessoas em geral. A possibilidade de transmissão pelo beijo de pessoas sob tratamento foi relatada por $86,5 \%$ dos participantes. Além disso, $85,3 \%$ dos entrevistados disseram que a transmissão é possível quando dormindo no mesmo quarto com pessoas com tuberculose durante esta fase do tratamento ${ }^{(15)}$.

$\mathrm{Na}$ análise bivariada, pode-se constatar que a renda mensal mais baixa esteve associada ao conhecimento, entretanto esperava-se que outros aspectos, como escolaridade e acesso aos meios de comunicação estivessem ligados ao conhecimento suficiente ou não das famílias pesquisadas sobre TB.

A renda abaixo de 1,7 salários mínimos mostrou-se correlacionada ao não conhecimento sobre TB. Para estes achados, levantou-se possíveis explicações, como alocação e distribuição das ESF em áreas populacionais com menor poder aquisitivo, ou seja, as pessoas com menor renda habitam na maioria das vezes lugares periféricos, onde estão localizadas as unidades básicas de saúde. E são nessas unidades que esses indivíduos encontram referência de informações relacionadas à sua patologia.

Em pesquisa realizada na capital do estado do Pará, os resultados mostraram que as informações transmitidas pelos profissionais aos clientes em suas consultas foram a principal fonte de informação sobre a doença, e essas, permeando seus conceitos pré-existentes, configuraram-se em um elemento importante na formação da representação da tuberculose como doença curável ${ }^{(16)}$.

Vale destacar que por se tratar de um estudo de amostragem por conveniência, não se pode 
confirmar com precisão esta associação, porque estes resultados podem trazer vieses do processo de seleção. Assim, sugere-se novos estudos, com plano amostral mais robusto, probabilístico com vistas à verificação da força entre o efeito de variáveis aleatórias - renda no conhecimento produzido pelas famílias sobre TB.

Como limitações do estudo, destaca-se que por ser uma investigação de corte transversal, há a possibilidade da existência de vieses comuns a este tipo de estudo, tais como o de informação e de memória. Além disso, o tamanho da amostra investigada pode impedir a generalização dos achados da pesquisa.

\section{- CONCLUSÃO}

O estudo evidenciou que o conhecimento expresso pelos familiares sobre a TB, em alguns aspectos foi demonstrado ser satisfatório. Entretanto, suscitou características preocupantes em relação a alguns fatores, tais como: a falta de interesse dos familiares em procurar informações sobre a tuberculose; o desconhecimento sobre o microrganismo responsável pela a doença; e a indicação da água e de alimentos contaminados como meios de disseminação da doença.

No que concerne aos fatores associados ao conhecimento sobre TB, constatou-se que os indivíduos com baixa renda apresentaram menor chance de ter conhecimento sobre a doença.

Sabe-se que o conhecimento sobre os mecanismos de transmissão, sintomatologia e tratamento medicamentoso, aliado às atitudes e comportamentos, opiniões e percepções a respeito da tuberculose, podem intervir em todo o processo de saúde, adoecimento e cura da doença. Deste modo, é imprescindível a promoção e disseminação da informação para complementar o conhecimento sobre a doença, o que pode ser obtido através dos diferentes setores da sociedade, da saúde, da educação, além da mídia.

Destarte, os achados deste estudo evidenciaram que as famílias ainda não estão sendo consideradas nas práticas de saúde da APS. Nesta perspectiva, levando em consideração as fragilidades relacionadas ao cuidado às famílias, recomenda-se aos profissionais que compõem a APS que as informações sobre a tuberculose sejam dadas de forma frequente, tanto ao doente, quanto aos seus familiares. É preciso, pois que a viabilização de estratégias de educação em saúde para esta parte da população seja parte integrante do tratamento da TB oferecido pelas unidades de saúde, assim como é preconizado pelo Ministério da Saúde.

\section{- REFERÊNCIAS}

1. Ministério da Saúde (BR). Secretaria de Vigilância em Saúde. Boletim Epidemiológico. Brasília [Internet] 2015 [acesso em 15 out 2015]. Disponível: http://portalsaude.saude.gov.br/images/pdf/2015/marco/27/2015-007---BETuberculose---para-substitui----o-no-site.pdf.

2. Paiva RS, Valadares GV, Pontes JS. A necessidade de tornar-se cuidador familiar: teoria fundamentada em dados. Online Braz J Nurs. [Internet] 2012; 11(3) [acesso em 14 jan 2014]. Disponível: http://dx.doi.org/10.5935/16764285.20120040.

3. Freitas IM, Crispim JÁ, Pinto IC, Villa TCS, Brunello MEF, Pinto PFPS, et al. Conhecimento e percepção sobre tuberculose das famílias de pacientes em tratamento diretamente observado em um serviço de saúde de Ribeirão Preto-SP, Brasil. Texto Contexto Enferm. [Internet] 2012; 21(3) [acesso em 30 nov 2014]. Disponível: http://dx.doi. org/10.1590/S0104-07072012000300020.

4. Corrêa GHLST, Bellato R, Araújo LFS. Networks to care woven by elderly and her family experiencing situations of chronic illness. Reme, Rev. Min. Enferm. [Internet] 2014; 18(2) [acesso em 12 dez 2015]. Disponível: http:// dx.doi.org/10.5935/1415-2762.20140027.

5. Alves PC. A experiência da enfermidade: considerações teóricas. Cad. Saúde Pública. [Internet] 1993; 9(3) [acesso em 30 out 2014]. Disponível: http://dx.doi.org/10.1590/S0102-311X1993000300014. 
6. Hoa NP, Chuc NTK, Thorson A. Knowledge, attitudes, and practices about tuberculosis and choice of communication channels in a rural community in Vietnam. Health policy. [Internet] 2009; 90(1) [acesso em 30 out 2014]. Disponível: http://dx.doi.org/10.1016/j.healthpol.2008.08.006.

7. Jittimanee SX, Nateniyom S, Kittikraisak W, Burapat C, Akksilp S, Chumpathat N, et al. Social stigma and knowledge of tuberculosis and HIV among patients with both diseases in Thailand. PLos ONE. [Internet] 2009; 4(7) [acesso em 29 out 2012]. Disponível: http://dx.doi.org/10.1371/journal.pone.0006360.

8. Abebe G, Deribew A, Apers L, Woldemichael K, Shiffa J, Tesfaye M, et al. Knowledge, health seeking behavior and perceived stigma towards tuberculosis among tuberculosis suspects in a rural community in southwest Ethiopia. PLos ONE. [Internet] 2010; 11(5) [acesso em 29 out 2012]. Disponível: http://dx.doi.org/10.1371/journal. pone.0013339.

9. Instituto Brasileiro de Geografia e Estatística (IBGE). Sistema IBGE de Recuperação Automatica (SIDRA). [Internet] Censo Demográfico 2010. [acesso em 29 out 2012]. Disponível: http://www.ibge.gov.br/home.

10. Freitas IM, Popolin MP, Touso MM, Yamamura M, Rodrigues LBB, Santos Neto M, et al. Fatores associados ao conhecimento sobre tuberculose e atitudes das famílias de pacientes com a doença em Ribeirão Preto, São Paulo. Rev. bras. epidemiol. [Internet] 2015; 18(2) [acesso em 12 dez 2015]. Disponível: http://dx.doi.org/10.1590/19805497201500020004.

11. Bowkalowski C, Bertolozzi MR. Vulnerabilidades em pacientes com tuberculose no Distrito Sanitário de Santa Felicidade - Curitiba, PR. Cogitare enferm. [Internet] 2010; 15(1) [acesso em 30 out 2012]. Disponível: http:// dx.doi.org/10.5380/ce.v15i1.17178.

12. Queiroga RPF, Sá LD, Nogueira JA, Lima ERV, Silva ACO, Pinheiro PGOD, et al. Distribuição espacial da tuberculose e a relação com condições de vida na área urbana do município de Campina Grande. Rev. bras. epidemiol. [Internet] 2012; 15(1). [acesso em 26 jun. 2015]. Disponível: http://dx.doi.org/10.1590/S1415790X2012000100020.

13. Sanchez AIM, Bertolozzi MR. Operacionalização do conceito de vulnerabilidade à tuberculose em alunos universitários. Ciênc. saúde colet. [Internet] 2011; 16(2) [acesso em 30 out 2012]. Disponível: http://dx.doi. org/10.1590/S1413-81232011000200031.

14. Ministério da Saúde (BR). Secretaria de Vigilância em Saúde. Departamento de Vigilância Epidemiológica. Manual de recomendações para o controle da tuberculose no Brasil. Brasília: Ministério da Saúde; 2011.

15. Mussi TVF, Traldi MC, Talarico JNS. Knowledge as a factor in vulnerability to tuberculosis among nursing students and professionals. Rev. esc. enferm. USP. [Internet] 2012; 46(3) [acesso em 12 nov 2012]. Disponível: http://dx.doi.org/10.1590/S0080-62342012000300023.

16. Rodrigues ILA, Souza MJ. Representações de clientes sobre a tuberculose: desvendar para melhor cuidar. Esc. Anna Nery. [Internet] 2005; 9(1) [acesso em 15 nov 2012]. Disponível: http://www.revistaenfermagem.eean.edu. br/audiencia_pdf.asp?aid2=938\&nomeArquivo=v9n1a11.pdf. 